\title{
NEW SOLUTIONS BASED ON WIRELESS NETWORKS FOR DYNAMIC TRAFFIC LIGHTS MANAGEMENT: A COMPARISON BETWEEN IEEE 802.15.4 AND BLUETOOTH
}

\author{
Mario Collotta', Giovanni Pau ${ }^{2}$ \\ Faculty of Engineering and Architecture \\ Kore University of Enna, Cittadella Universitaria, 94100 Enna, Italy \\ E-mails: ${ }^{1}$ mario.collotta@unikore.it; ${ }^{2}$ giovanni.pau@unikore.it
}

The Wireless Sensor Networks are widely used to detect and exchange information and in recent years they have been increasingly involved in Intelligent Transportation System applications, especially in dynamic management of signalized intersections. In fact, the real-time knowledge of information concerning traffic light junctions represents a valid solution to congestion problems. In this paper, a wireless network architecture, based on IEEE 802.15.4 or Bluetooth, in order to monitor vehicular traffic flows near to traffic lights, is introduced. Moreover, an innovative algorithm is proposed in order to determine dynamically green times and phase sequence of traffic lights, based on measured values of traffic flows. Several simulations compare IEEE 802.15.4 and Bluetooth protocols in order to identify the more suitable communication protocol for ITS applications. Furthermore, in order to confirm the validity of the proposed algorithm for the dynamic management of traffic lights, some case studies have been considered and several simulations have been performed.

Keywords: wireless sensor networks; road monitoring; traffic lights; intelligent transportation systems

\section{Introduction}

The increasingly growth of vehicles number requires the use of intelligent control systems in order to improve the livability of roads and reduce, as much as possible, accidents. Most of the accidents on the roads occur today because drivers are not aware of upcoming traffic hurdles, like traffic signals, curves and railway lines and so on. Another important issue to be discussed is represented by traffic lights. Accidents, at road intersections, occur for several reasons including the lack of care and the wrong management of traffic lights. The real-time knowledge of road information represents a valid solution to these problems with the main aim to reduce, as much as possible, accidents. The Intelligent Transportation Systems (ITS) aim to improve traffic quality by enhancing safety (Sànchez et al., 2013) through the reduction of travel time (Kothuri et al., 2006) and, as a consequence, of fuel consumption (Rommerskirchen et al., 2013) through gathering, organizing, analyzing, using, and sharing traffic information. Although many researchers focused on these issue, today most of the adopted solutions are very expensive and difficult to install and manage (Fei-Yue Wang, 2010). One of the main aspects on which investigate is represented by the communication medium used to exchange the gathered road information (Skordylis and Trigoni, 2011). Usually, the devices used to gather road data send the information to the central entity, or to a neighbor device, through the use of wired connections. Today, many researchers of different fields, discuss about the possibility to replace, totally or at least partially, the wired networks with the wireless (Eng-Han Ng et al., 2008) ones for these reasons:

- $\quad$ low cost of wireless devices (Gallart et al., 2011);

- $\quad$ ease of installation (Bee-Lie Chai et al., 2010);

- flexibility (Philipp et al., 2012): wireless networks can be used, at the same time, for several purposes and, thanks to the possibility to create several class of services, it is possible to properly manage data traffic flows based on their real time constraints;

- fault tolerance (Pileggi et al., 2010): the failure of one or more network nodes does not affect the functioning of the entire network;

- $\quad$ scalability (Alazzawi et al., 2008): to add or remove a network node is quite simple.

Moreover, wired networks need the power grid to work. As a consequence, it may be really difficult to install these systems in areas hard to reach or where there is no power available. These drawbacks are avoided by the evolution of embedded systems, wireless technologies and Wireless Sensor Networks 
(WSNs), characterized by their low size, ease of installation and thanks to their wireless communication they offer scalability with lower costs. Several works in literature discussed the manifold advantages provided by the use of wireless technologies in ITS scenarios (Collotta et al., 2015), such as the reduced costs of installations (Huck et al., 2005), better flexibility, the ability to easily realize temporary deployments (e.g. for monitoring purposes), etc.. ITS applications can be categorized depending on many factors:

- drivers' safety by transmitting accidents and weather information;

- traffic management to minimize congestion or traffic jam and optimize road capacity utilization. This category includes traffic optimization by applying automated traffic assistance decisions and real-time traffic light handling in both isolated and interconnected intersections;

- $\quad$ smart cities. This category includes:

o navigation orientations before and during a trip in order to minimize cost;

o pollution prevention;

o parking locations finding and optimization;

o road maintenance and repair;

o public transports management;

o electronic transactions to allow fees payment on roads and parking;

o Vehicle To Vehicle communications (V2V).

It is possible to distinguish the main fundamental requirements that should be fulfilled by any ITS technology:

- data reliability: it is of high importance for urban traffic monitoring and management, but this becomes challenging when using WSN due to the low coverage and limited capacity of sensor nodes, added to the "lossy" nature of wireless communication channels. Therefore, ensuring reliable end-to-end communication must be taken into account by any proposed WSN architecture and communication protocols;

- communication security: it must be efficiently handled by appropriate solutions because wireless nature of communications in WSN is more prone to security attacks than wired technologies. The wireless devices may be deployed without physical protection or surveillance and may also store sensitive information relating to users privacy. Therefore, traffic management solutions based on WSN must take into consideration the vulnerable nature of WSN and their components. Specifically, the communication protocols must be secure in order to prevent attacks and sufficiently reliable and fault tolerant to guarantee service continuity in case of failures;

- interoperability and scalability: the rapid development of wireless technologies and different types of sensors imposes on the proposed solutions to take into account hardware heterogeneity to ensure interoperability and scalability;

- real-time communication: many applications need real-time information collection to take intime decisions. Traffic light monitoring is a typical example, where dynamic crossroad management depends on real-time traffic distribution.

All of these issues can be addressed through innovative ideas that may individually, or in a complementary manner, cover the following aspects:

- design of multi-tiered network architectures: often it is necessary to organize the road areas to be monitored in cells. As a consequence, it is really important to provide mechanisms and protocols in order to ensure the interconnections among cells. Another aspect to consider is represented by the need to ensure the possibility to realize systems aimed to ease the integration of different devices designed to work for specific and different tasks;

- implementation of new mechanisms, or protocols, in order to improve/ensure the reliability of communications on ITS solutions;

- development of techniques to support the transmission of real time constrained data;

- design of decision making approaches based on soft-computing solutions: the management of vehicular traffic flows or traffic lights is a problem often manageable by natural languages. A possible starting point could be represented by new soft computing techniques to improve or re-design aspects of neural networks or fuzzy logic traditionally used by researchers in these fields; 
- design and implementation of secure biometric access techniques to ensure the security of personal data that can be stored on the devices.

This work shows a novel network architecture for dynamic management of signalized intersections Specifically, this paper shows an implementation of this approach both on IEEE 802.15.4 (802.15.4, 2006) and on Bluetooth (Bluetooth, 2010) networks in order to determine the best technology meeting the requirements that characterize road monitoring environments. Moreover, in this paper is also proposed an algorithm for the dynamic management of traffic lights. The paper is organized as follows. Section 2 describes main related works in order to determine the current state of art. Section 3 describes the network architecture and the proposed approach showing its implementation both in IEEE 802.15.4 and in Bluetooth networks. Section 4 presents a simulation scenario in order to show network performance obtained by the proposed approach in both network technologies. Finally, Section 5 summarizes the paper reporting conclusions.

\section{Related Works}

\subsection{IEEE 802.15.4 for ITS applications}

The management of signalized intersections through the use of IEEE 802.15.4 has been presented in several works in the literature. An intelligent traffic congestion monitoring and measurement system in order to monitor and measure the road traffic congestions is presented by Mandal et al. (2011). The authors propose a rapidly deployable, cost-effective and easily maintainable system that is based on IEEE 802.15.4 protocol and GSM technologies. The proposed approach is based upon calculation of vehicular speed over a stretch of road and the average waiting time of vehicles at road-crossing. After analyzing other technologies both on the market and in the literature, the authors show the obtained results and these are promising.

In another literature work (Zotos et al., 2012) the results of a case study focused on an experimental installation of an intelligent road lighting system are presented. The authors introduces an energy-efficient, intelligent outdoor lighting management and monitoring system where data communication is based on wireless personal area networks. The proposed system maintains lighting at a low level until vehicle or pedestrian motion is detected by a set of proximity sensors. In this case, the level of lighting is increased in order to provide better visibility while the area is occupied and dims to a lower level when the area is clear again. At the same time, data regarding lamp parameters like status and operation dimming level are transferred to the management system via a bidirectional channel that may receive feedback based on a certain system operation profile. Experimental results show that using the proposed system $37 \%$ of energy can be saved without causing problems or disturbing daily routing and safety. The combination of communication based on new wireless technologies can be an energy saving and environmentally-friendly solution.

A comprehensive and thorough analysis of implementing a WSN in ITS is presented by Megalingam et al. (2010). Two practical applications of WSN in this context have been identified and implemented by the authors with extensive description of the software and hardware implementation process. In detail, a smart vehicle speed monitoring and traffic routing system is proposed using WSNs in order to monitor and report about the speeding vehicles and also to regulate the traffic. Experimental results reveal that the proposed system is highly flexible and adaptive to traffic conditions. Moreover, the system proposed by the authors is comparatively more economical when compared to the conventional advanced techniques involved in modem ITS due to lower deployment and maintenance costs.

Brahmi et al. (2013) introduce a new adaptive backoff scheme for IEEE 802.15.4 protocol, in order to ensure faster transmission of emergency messages in road environment. The main aim of the authors is to adapt the backoff selection interval to the class of message being transmitted based on a normal distribution. Using the proposed approach, the messages reporting incidents or dangerous events are granted a smaller backoff compared to those carrying periodic traffic flow or weather information. The performance evaluation results, obtained varying both WSN size and periodic messages transmission frequencies, highlight the effectiveness of the proposed approach.

A fuzzy logic controller, for a flexible Quality of Service (QoS) management, is presented by Collotta et al. (2012). The authors show an innovative WSN architecture, based on IEEE 802.15.4 protocol, and a novel algorithm that dynamically enables/disables some video cameras according to the real need to monitor a given area. The algorithm is based on measured traffic volume values through the WSN. The possibility to activate more devices only in case of real need, ensures energy savings. Furthermore, using a 
fuzzy logic controller, the proposed approach allows to manage easily and in a better way network workload changes.

\subsection{Bluetooth for ITS applications}

Bluetooth networks are used in several applications for their useful features. The possibility to use real- time information in order to dynamically manage the traffic light cycles is a key aspect of ITS applications. Collotta et al. (2014) propose a Bluetooth network architecture in order to monitor vehicular traffic flows near to a traffic light. The proposed architecture is characterized by a novel algorithm in order to determine green times and phase sequences of traffic lights, based on measured values of traffic flows. However, Bluetooth sensor devices are battery powered, whereby batteries must be replaced after their normal duration (dependent on the use). For this reason the proposed approach is based on real time information provided by a Bluetooth sensor network powered by energy harvesting devices inserted within a road cavity in order to catch the external vibrations, caused by the passage of vehicles. Several simulations have been carried out and obtained results are very promising both in terms of queues management and in terms of the proposed energy harvesting technique.

The travel time of signalized arterial routes using Bluetooth data is analyzed by Tsubota et al. (2011). This data is gathered from scanners, located in signalized arterial routes, when Bluetooth devices pass through the detection range. The analysis carried out by the authors confirms that travel time obtained from Bluetooth records captures traffic characteristics in urban arterial streets, such as morning and evening peaks during weekdays, and bi-modal travel time in a particular link.

Jie et al. (2011) propose a method to measure or estimate the travel time in urban road networks. The authors highlight that urban travel times display a large variation, so that the measurement of a single (average) travel time is not so meaningful. So, the travel time distribution is more relevant than the single value of the average. This distribution can be obtained from observations of travel times of individual vehicles. The distribution of travel time can be measured by Bluetooth scanners that can recognize Bluetooth devices in cars. The authors point out some issues after installing the Bluetooth scanner. In fact, the devices with Bluetooth are often mobile telephones that can be carried by pedestrians, cyclists and passengers of public transport. It is possible that some vehicles have several Bluetooth devices on board. This causes a bias in the estimation of travel time distributions. Furthermore, the exact position where the Bluetooth devices are registered cannot accurately be determined. For longer distance applications like on freeways, such an uncertainty has a smaller impact, while on urban roads the relative impact can be large.

In another literature work (Araghi et al., 2013) an accurate travel time estimation and incident detection using Bluetooth and Wi-Fi technologies is presented. After a through analysis, the authors came to the conclusion that Bluetooth sensors can be considered as a cost-effective method for expanding the monitoring capabilities on a wider road network and providing real time information of the traffic condition both for the traveler's information systems and traffic management systems. Moreover, the Bluetooth sensor design and configuration of antennae could play a significant role in improving the capability of the proposed system and expanding its application on various traffic monitoring aspects such as incident detection and queue analysis.

The main aim of Bachmann et al. (2014) is to combine a Bluetooth traffic monitoring system with loop detector data for improved freeway traffic speed estimation. Several approach are shown and they are implemented and compared in terms of their ability to fuse data from loop detectors and probe vehicles to accurately estimate freeway traffic speeds. Data from a Bluetooth traffic monitoring system are fused with corresponding loop detector data and compared against GPS collected probe vehicle data. The analysis shows that through data fusion, even a few probe vehicle measurements from a Bluetooth traffic monitoring system can improve the accuracy of traffic speed estimates traditionally obtained from loop detectors.

\section{Wireless Network-based solution for traffic light management}

\subsection{System architecture}

The proposed architecture, depicted in Figure 1, is a hierarchical network characterized by two layers: one layer is represented by a wireless sensor network, based on the IEEE 802.15.4 or Bluetooth protocols, while the other one consists of a wired backbone. The IEEE 802.15.4 protocol $(802.15 .4,2006)$, in beacon-enabled mode, makes possible an a priori scheduled communication using Guaranteed Time Slot (GTS). The use of GTS allows to have a communication as deterministic as possible. The WSN architecture here proposed is based on the GTS mechanism. 


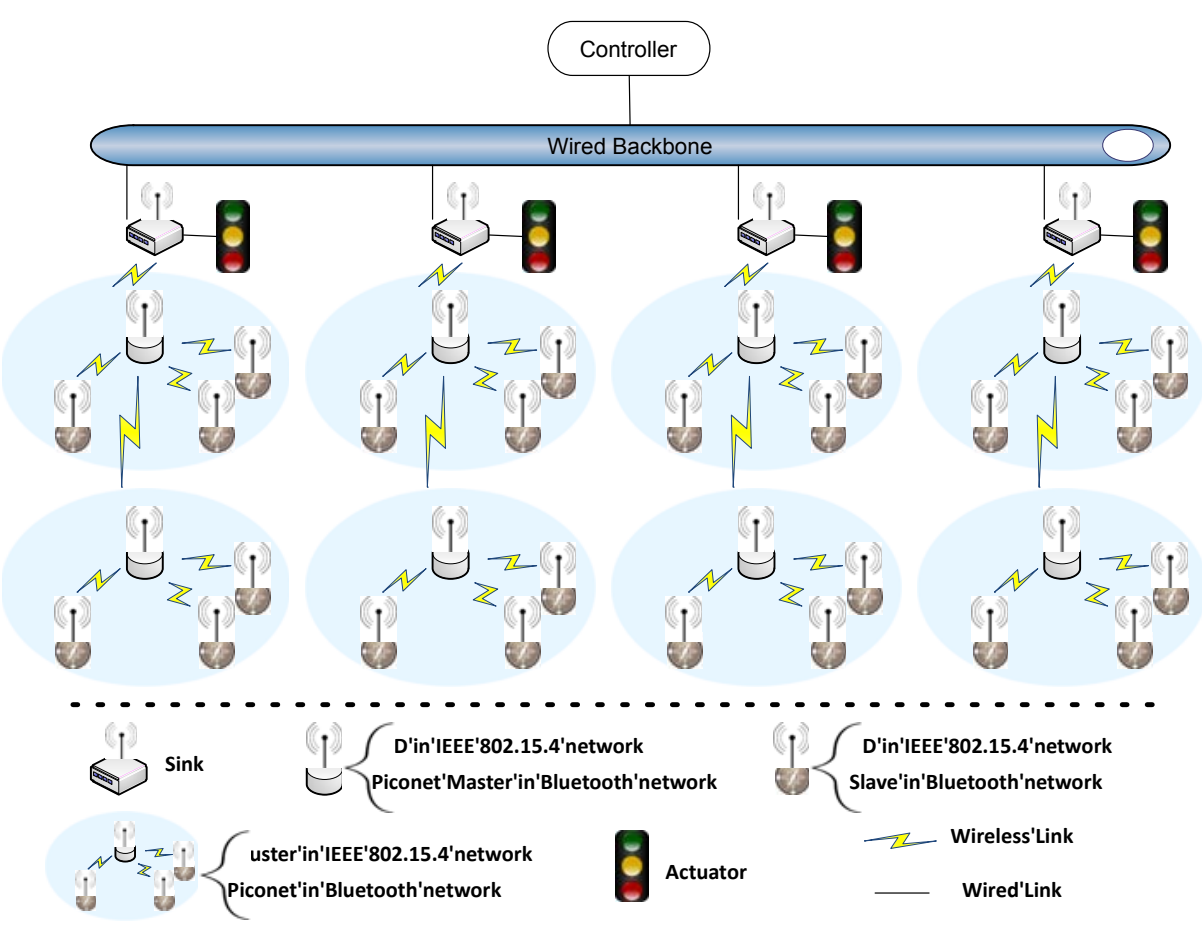

Figure 1. Network architecture

The designed two-tiered architecture is valid for a generic road intersection. Considering the IEEE 802.15.4 protocol, for each intersection several RFD (Reduced Function Device) sensor nodes, organized in clusters and coordinated by special FFD (Full Function Device) nodes, are used. The whole network is coordinated by a sink node that collects and processes all data concerning vehicles. All sink nodes are connected through a wired backbone to a central controller. Each IEEE 802.15.4 cluster consists of a FFD node and several RFD nodes. The FFD node is an 802.15.4 cluster coordinator and it also takes care of routing among clusters. The communication among nodes of the same sub-network is realized using GTS. Periodically, the FFD node sends a data request to its RFD nodes. These nodes reply to the request by sending collected data. Each RFD node is able to detect vehicles in transit, using magnetometers. Thanks to the use of beacons, nodes may enter in sleep mode and save energy during inactivity periods, while waiting for next beacon.

Regarding to Bluetooth (Bluetooth, 2010), it is important to underline that it does not natively support real-time communications. As demonstrated by Collotta et al. (2013), a deadline-aware scheduling should be introduced in order to ensure the satisfaction of real-time constraints (Collotta et al., 2007). Anyhow, the architecture proposed in this paper is based on the standard Bluetooth protocol, without hardware or software changes. In fact, according to Bluetooth standard, a Piconet is formed by a Master node and a maximum of 7 Slaves. If the network requires more than 7 slaves then it is possible to implement a Scatternet, a type of ad-hoc computer network consisting of two or more Piconets. Scatternets can be formed when a member of one Piconet participates as a slave in another Piconet. This Slave transmits data among members of both networks (Collotta et al., 2010). In the Bluetooth approach, the traffic light junctions are monitored using appropriate sensors nodes, (Slaves) and the data gathered by them is then forwarded to their Piconet Master which collect information of the Piconet and forward them to the sink which takes care of data exchanging between Piconets.

As shown in Figure 1, each approach is provided with a sink node, which performs several tasks described below. Specifically, the Scheduling module manages networks real-time traffic, allows bandwidth reservation and avoids collisions using a real-time algorithm, e.g. Earliest Deadline First (Horn, 1974). The Error Handling module controls transmission errors. It works in cooperation with the Scheduling module, communicating the presence of errors, and it adapts the data scheduling to ensure continuous flow of traffic. The Resources Monitoring module manages and shares the availability of resources, e.g. by periodically assessing the channels quality. The Actuator Interface module manages communication with 
the actuator, by sending commands for the timing of lighting and shutdown of the traffic lights optical units. At periodic intervals, the sink node receives data from sensors and sends them via the wired backbone to the controller.

The controller is characterized by several modules in order to realize the data collection, the data processing and the traffic lights control. The Data Collection module receives and organizes data sent by sink nodes. Data traffic is stored into a database, to allow future referencing for all measurements. The Processing module reads data organized by Data Collection module and processes them trough an appropriate algorithm. This algorithm manages the received data in order to determine the phase sequence and the green time of each phase. Parameters like length of the queue for various lanes and the number of vehicles in transit are used by the algorithm. Finally, the Traffic Lights Control module uses the results produced by the algorithm in order to manage the sequence and the duration of traffic light phases, by sending commands to the sink. These data are then forwarded to the actuator.

\subsection{Dynamic traffic light manager algorithm}

The dynamic traffic light management allows runtime and real-time regulation of phase sequence and green times duration, based on data gathered by a WSN deployed on the road. The proposed dynamic management algorithm is divided into two steps. In the first step the phase sequence is deter- mined. In fact, the algorithm, based on the queue length for each flow (input variable), assigns a priority to each phase equal to the maximum queue length of that phase. Finally, it determines the phase sequence by sorting them in descending order priority. The second step realizes the green times calculation. The algorithm, for each flow, processes the number of vehicles passed during the previous traffic light cycle and uses this value to determine the current traffic volume $\left(Q_{-} R T\right)$. This value is used to recalculate the green time duration of the next cycle based on traffic detected. The Figure 2 shows in detail green times calculation flowchart.

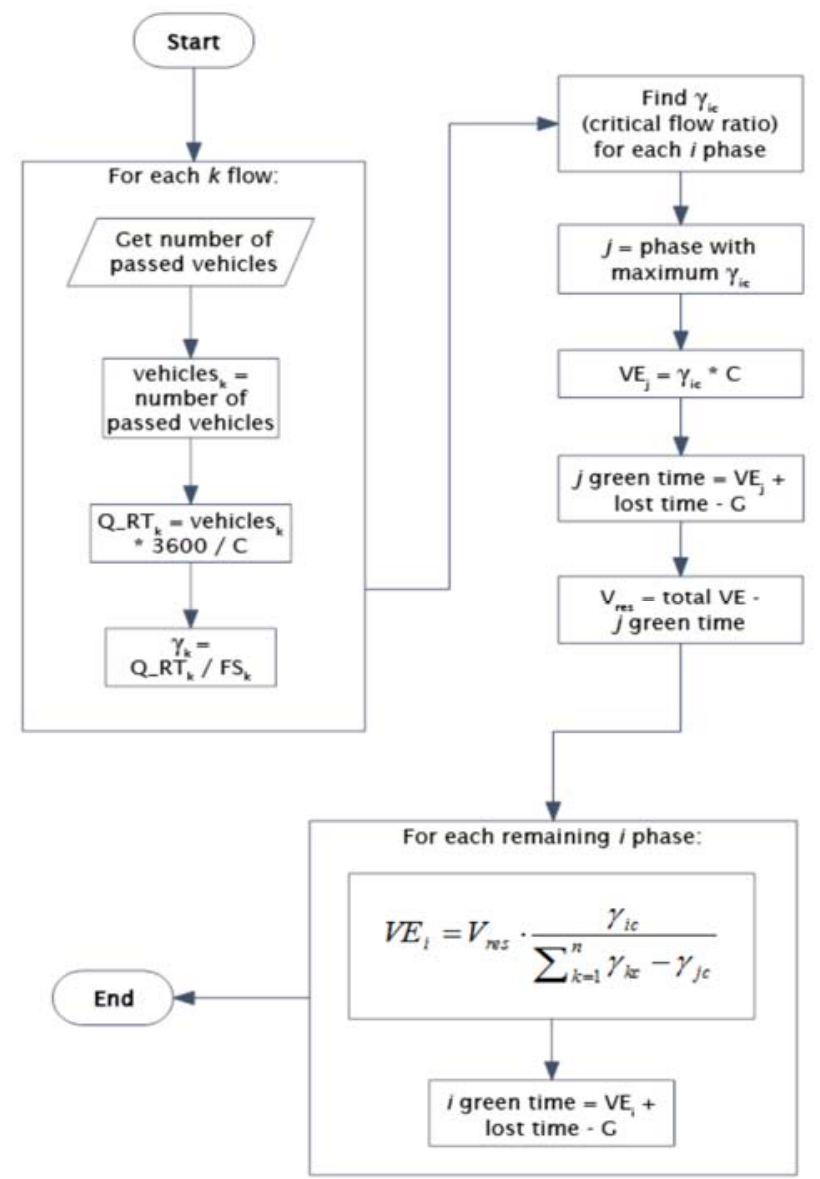

Figure 2. Green times calculation flowchart 
The flow ratio $\gamma$ for a generic flow $i$ is defined as the ratio between the actual or expected incoming stream $Q$ (or volume) and the saturation flow rate $F S$ :

$\gamma_{i}=\frac{Q_{i}}{F S_{i}}$

Data coming from WSN is used to recalculate the incoming stream at every traffic light cycle. For each lane, the number of vehicles passed in each cycle is counted. This data is converted in vehicles/h and subsequently used to recalculate the flow ratio for each traffic flow. For each phase, the critical lane (the one with a higher flow ratio) is established. The phase with the highest flow ratio is found; to this $j$-phase we assign a saturation flow ratio equal to 1, giving rests to the remaining phases in proportion to their flow ratios:

$\frac{V E_{j}}{C}=\gamma_{i}$

where $V E_{j}$ is the effective green time for the $j$-phase and $C$ is the traffic light cycle duration. Once $V E_{j}$ is obtained, it is possible to calculate the $j$-phases $V$ green time using the following equation:

$V_{j}=V E_{j}+P-G$,

where $P$ indicates lost time due to start-up and permission times and $G$ indicates the yellow time. The seconds available for remaining phases are calculated as:

$V_{\text {res }}=\sum_{j=1}^{n} V E_{j}-V_{j}$

For remaining green time $\left(V_{\text {res }}\right)$ allocation, the following equation can be used:

$V E_{i}=V_{\text {res }} \frac{\gamma_{i c}}{\sum_{k=1}^{n} \gamma_{k c}-\gamma_{j c}}$

Finally, the green time for each $i$-phase is calculated using the equation (3).

\section{Performance Evaluation}

In order to validate the benefits introduced by the proposed approach considering both IEEE 802.15.4 and Bluetooth, several simulations have been carried out. Regarding to IEEE 802.15.4, the simulations have been conducted using OMNeT++ (OMNeT++, 2014) considering several cluster topologies consisting of a Full Function Device (FFD) and seven end devices (RFD). The same topology has been used for measurements concerning a Bluetooth network, a Master and seven Slaves forming a Piconet. In this case, simulations have been carried out through the ucbt extension of NS-2 simulator (Network Simulator, 2013). In both cases, the Throughput/Workload (Th/Wl) percentage, the deadline miss ratio, and delays related to both periodic and aperiodic flows have been measured considering packet size of $18 \mathrm{~KB}$ and data rate of $180 \mathrm{kbps}$ for each station. It is useful to note that the Throughput is the sum of packets sent by the device and the Workload is the total number of packets that the device has to send. The periodic flows (packets) refer to the monitoring of network resources and to the data collected by sensor nodes. On the contrary, aperiodic flows deal with the error handling and the scheduling.

Figure 3 shows a comparison between results obtained in IEEE 802.15.4 and Bluetooth, respectively. It is possible to see that in the IEEE 802.15.4 network produces better performance. Periodic $\mathrm{Th} / \mathrm{Wl}$ percentage measured is $81.7 \%$ against $67.2 \%$ measured in a Bluetooth scenario, while aperiodic $\mathrm{Th} / \mathrm{Wl}$ percentage obtained is $16.3 \%$ against $21.8 \%$ obtained in a Bluetooth scenario. 


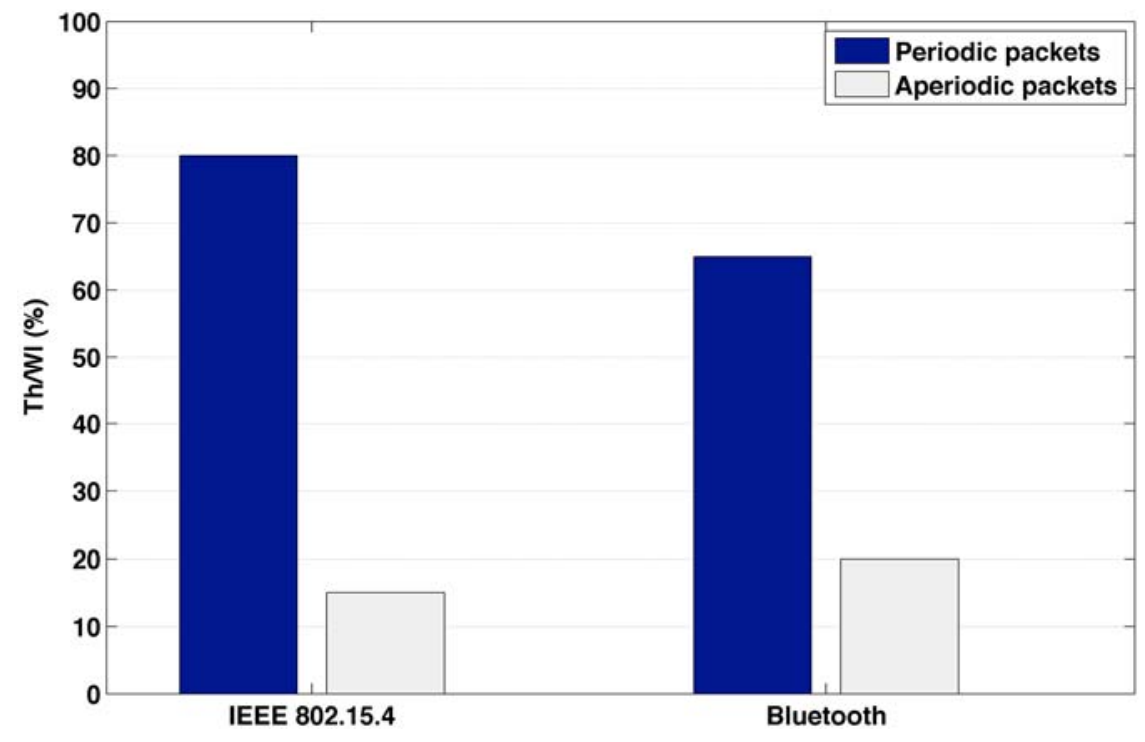

Figure 3. Th/Wl behavior comparison

Deadline miss ratio results in IEEE 802.15.4 and Bluetooth, respectively, are depicted in Figure 4. In an IEEE 802.15.4 network less deadline miss percentage has been measured than Bluetooth for both periodic (4.1\% versus $6.4 \%)$ and aperiodic traffic flows (1.9\% versus $2.1 \%)$.

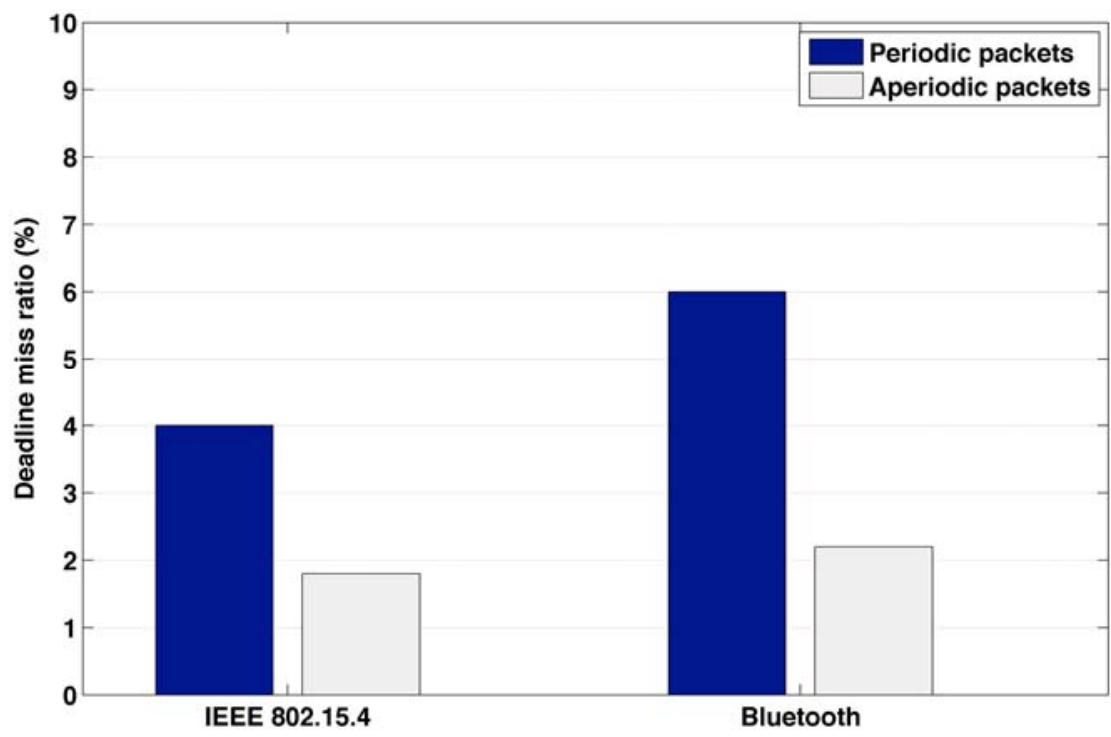

Figure 4. Deadline miss ratio comparison

Finally, Figures 5 and 6 show average delay for periodic and aperiodic packets, respectively. It is possible to see that Bluetooth produces on average a delay of $31.43 \mathrm{~ms}$ lower than IEEE 802.15.4 in case of periodic traffic flows while, in case of aperiodic traffic flows, the estimated delay is on average 19.21 ms lower using a Bluetooth network. 


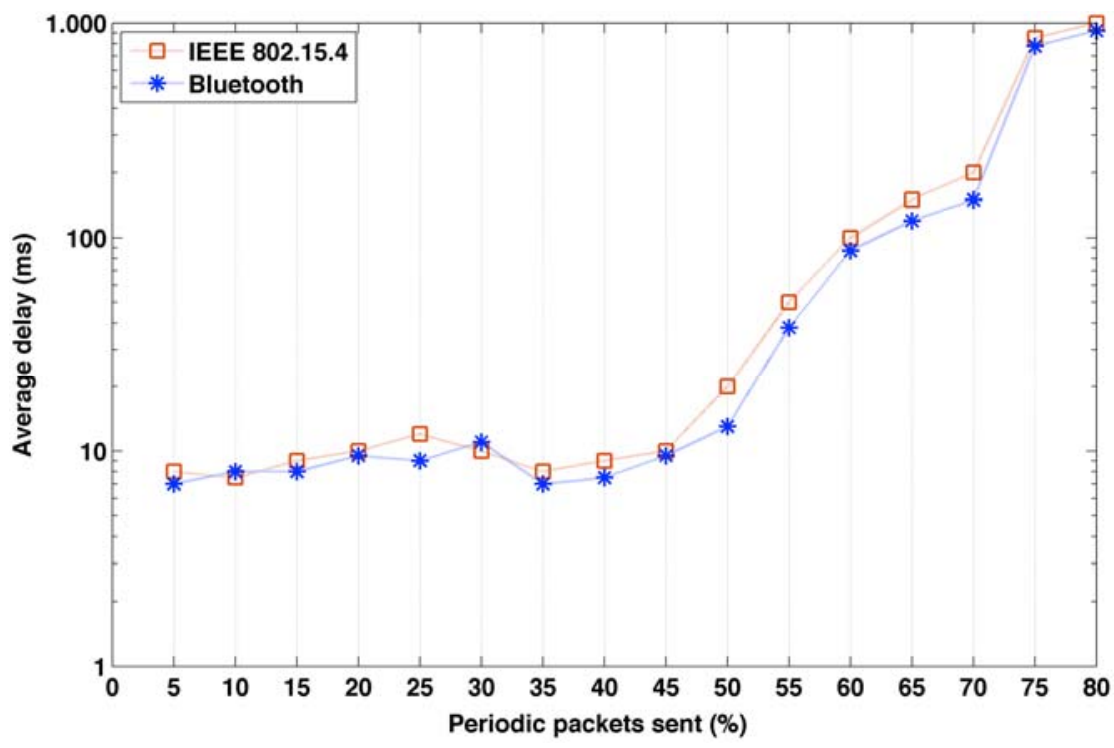

Figure 5. Average delay for periodic packet

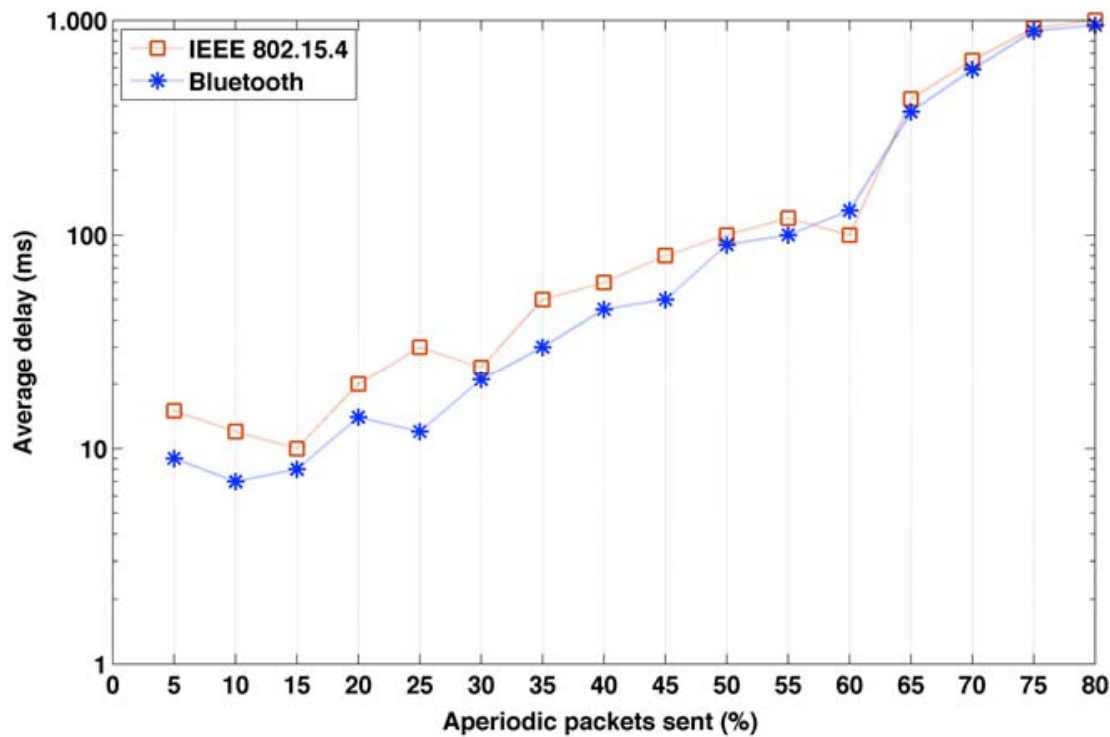

Figure 6. Average delay for aperiodic packet

Furthermore, other simulations have been carried out in order to determine the performance of the algorithm for the dynamic management of the traffic light. The simulations have been carried out both in case of fixed and dynamic traffic light cycle in order to demonstrate the goodness of the proposed approach. Each simulation provides average queue length trend for traffic light cycles. Traffic volume levels set in the different case studies are the following:

- $\quad$ simulation $1=$ low traffic volume;

- $\quad$ simulation 2 = medium traffic volume;

- $\quad$ simulation 3 = high traffic volume.

Obtained results, depicted in Figure 7, show a reduction of average queue length in each case study with dynamic management. 


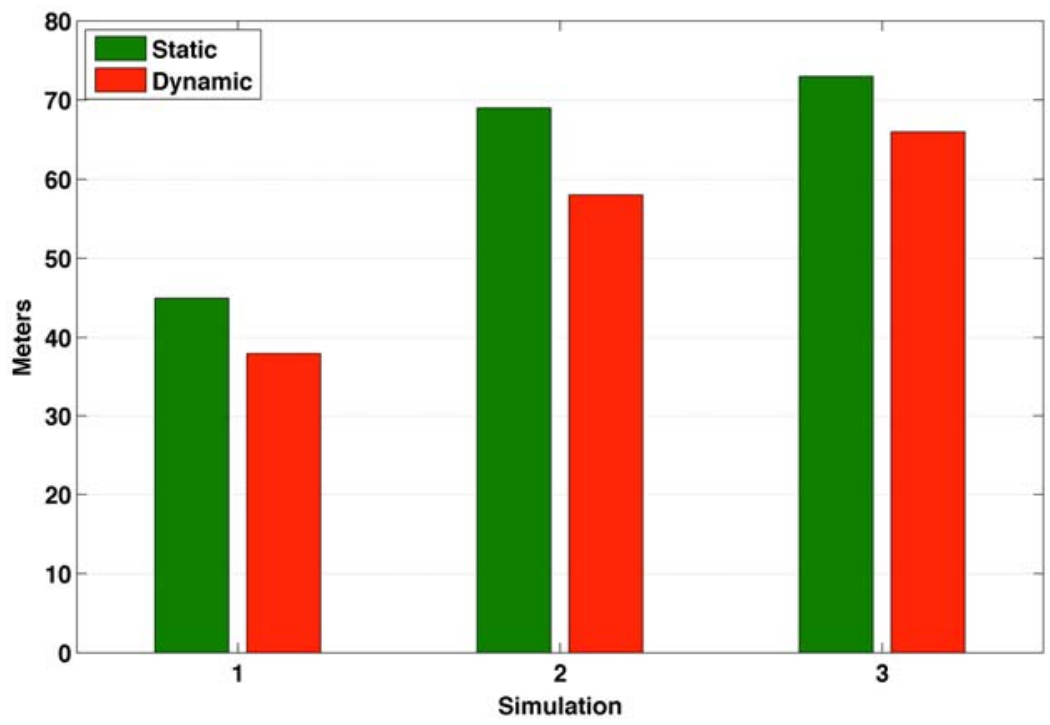

Figure 7. Average queue length: comparison between static and dynamic management

Also, it can be useful to associate the value of average queue length - $q_{a l}$ - with the value of junction approaches flow rate - $Q_{t}$ - and their relationship with the total number of vehicles crossing the intersection in an hour $-Q_{c}$ (see Table 1). It is helpful to highlight that the $Q_{c} / Q_{t}$ ratio between static and dynamic management is quite steady because of the cycle traffic light duration (and therefore the actual green) has been unchanged in both management.

Table 1. Comparison of $Q_{c} / Q_{t}$ between Static (S) and Dynamic (D) Management

\begin{tabular}{|l|l|l|l|l|l|l|l|}
\hline \multirow{2}{*}{ Simulation } & \multirow{2}{*}{ Overall flow rate $\left(Q_{t}\right)$} & \multicolumn{2}{|l|}{$q_{a l}$} & \multicolumn{2}{l|}{$Q_{c}\left(v e h_{p}\right)$} & \multicolumn{2}{l|}{$Q_{c} / Q_{t}$} \\
\cline { 3 - 8 } & & S & D & S & D & S & D \\
\hline 1 & & & & & & & \\
\hline 2 & 6600 & 45.14 & 37.67 & 6491 & 6509 & 0.984 & 0.986 \\
\hline 3 & 6600 & 69.82 & 58.83 & 6432 & 6458 & 0.975 & 0.979 \\
\hline
\end{tabular}

To understand in detail benefits obtained with traffic light dynamic management, Figure 8 compares peaks of average queue length. It has been observed that for some case studies the difference between static and dynamic management is greater while in other cases it is smaller. It depends on traffic volume entering the intersection from each approach. Because of values considered, it has been found that the algorithm has greater efficiency when the gap between traffic volumes coming from different intersections is higher. The algorithm manages these situations balancing green times according to traffic volume information coming from sensors. There are some improvements even if traffic level is not high, although to a lesser degree. This is due to green times recalculation based on actual traffic conditions. The ability of the designed WSN to fit traffic light phases in order to rebalance the relationship between crossing flows and queued flows, makes the results achieved so far particularly interesting, both in terms of total node efficiency (reduction of the average queue length value) and in terms of penalized / smoother maneuvers selection (transition of queue peaks among different entering maneuvers). 


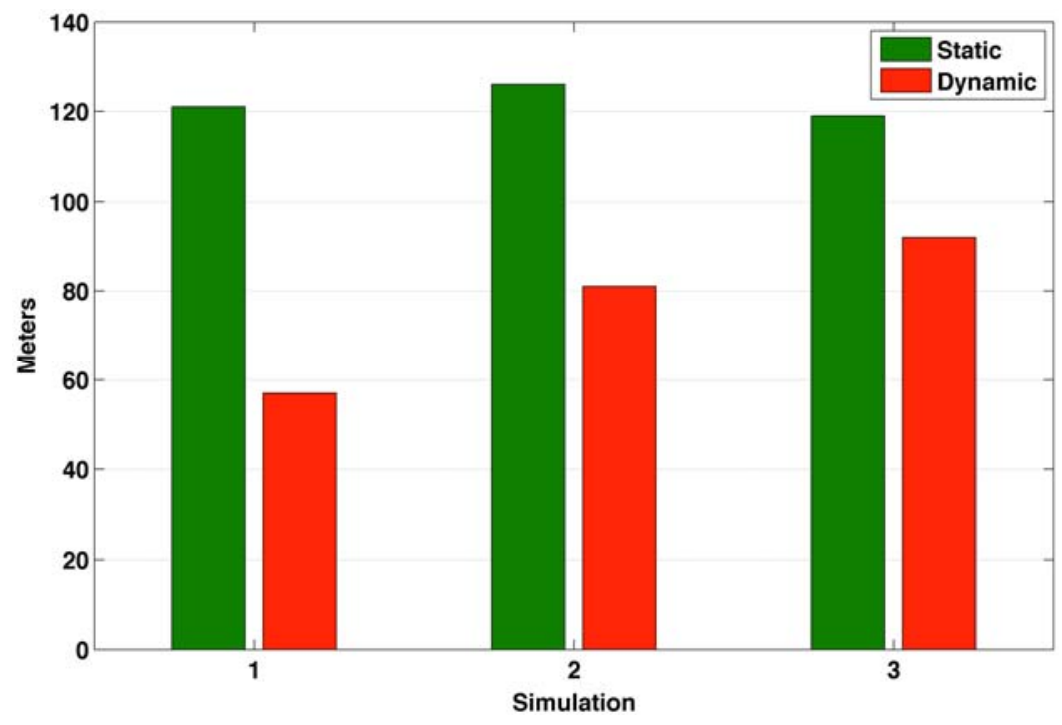

Figure 8. Peaks of average queue length: comparison between static and dynamic management

\section{Conclusions}

In this paper a Wireless Sensor Network architecture, with multiple levels and based on IEEE 802.15.4 or Bluetooth protocols, for traffic flows monitoring has been proposed. A comparison between IEEE 802.15.4 and Bluetooth solutions has been conducted in order to identify the more suitable communication protocol for ITS applications. The simulation results show that both wireless protocols behave well enough in the Intelligent Transportation System context. In fact, the $\mathrm{Th} / \mathrm{Wl}$ percentage of periodic flows measured in a IEEE 802.15 .4 network is $81.7 \%$ against $67.2 \%$ measured in a Bluetooth scenario. Similarly, better performance has been obtained in terms of deadline miss ratio for both periodic $(4.1 \%$ versus $6.4 \%)$ and aperiodic $(1.9 \%$ versus $2.1 \%)$ flows. On the contrary, in terms of average delay measured, Bluetooth produces better performance than IEEE 802.15.4. In fact, the measured delay of periodic traffic flows in Bluetooth is $31.43 \mathrm{~ms}$ lower than IEEE 802.15.4 while, in case of aperiodic traffic flows, the estimated delay in Bluetooth is on average 19.21 ms lower than IEEE 802.15.4. Considering these results, in applications where the main requirement is to have low packet loss reducing, at the same time, the deadline miss, it is preferable to use IEEE 802.15.4. On the contrary, in applications where it is important to ensure low network communication latencies, it is preferable to use Bluetooth.

Moreover, in this paper an algorithm for the dynamic management of intersections that use data collected by the WSN, to determine the phase sequence and the green times duration, has been implemented. The obtained results illustrate that using the implemented algorithm it is possible to achieve a better management of isolated traffic light junctions.

Regarding future intelligent system improvement, we are working on the improvement of the algorithm and on the optimization of the proposed network architecture. Furthermore, it could be inserted a priority system for emergency vehicles, as well as a preferential system for public transportation vehicles.

\section{References}

1. Sánchez, N.; Alfonso, J.; Torres, J.; Menéndez, J.M. (2013) ITS-based cooperative services development framework for improving safety of vulnerable road users. Intelligent Transport Systems, IET, vol.7, no.2, pp. 236-243.

2. Kothuri, S.M.; Tufte, K.; Soyoung Ahn; Bertini, R.L. (2006) Development of an ITS data archive application for improving freeway travel time estimation. IEEE Conference on Intelligent Transportation Systems (ITSC '06), pp.1263-1268.

3. Rommerskirchen, C.; Helmbrecht, M.; Bengler, K. (2013) Increasing complexity of driving situations and its impact on an ADAS for anticipatory assistance for the reduction of fuel consumption. IEEE Intelligent Vehicles Symposium (IV), pp.573-578.

4. Fei-Yue Wang (2010) Parallel Control and Management for Intelligent Transportation Systems: Concepts, Architectures, and Applications. IEEE Transactions on Intelligent Transportation Systems, vol.11, no.3, pp.630-638. 
5. Skordylis, A; Trigoni, N. (2011) Efficient Data Propagation in Traffic-Monitoring Vehicular Networks. IEEE Transactions on Intelligent Transportation Systems, vol.12, no.3, pp.680-694.

6. Eng-Han Ng; Su-Lim Tan; Guzman, J.G. (2008) Road traffic monitoring using a wireless vehicle sensor network. International Symposium on Intelligent Signal Processing and Communications Systems (ISPACS 2008), pp.1-4.

7. Gallart, V.; Felici-Castell, S.; Delamo, M.; Foster, Andrew; Perez, J.J. (2011) Evaluation of a Real, Low Cost, Urban WSN Deployment for Accurate Environmental Monitoring. IEEE 8th International Conference on Mobile Adhoc and Sensor Systems (MASS), pp.634-639.

8. Bee-Lie Chai; Ki-One Yi; Hyotaek Lim; Gwang-Hoon Kwark (2010) The implementation and performance evaluation of link power control in wireless sensor network. 2nd International Conference on Computer Engineering and Technology (ICCET), vol.6, pp.V6-245,V6-248.

9. Philipp, F.; Ping Zhao; Samman, F.A; Glesner, M.; Dassanayake, K.B.; Maheswararajah, S.; Halgamuge, S. (2012) Adaptive wireless sensor networks powered by hybrid energy harvesting for environmental monitoring. IEEE 6th International Conference on Information and Automation for Sustainability (ICIAfS), pp.285-289.

10. Pileggi, S.F.; Palau, C.E.; Esteve, M. (2010) Multimode WSN: Improving Robustness, Fault Tolerance and Performance of Randomly Deployed Wireless Sensor Network. Second International Conference on Computational Intelligence, Communication Systems and Networks (CICSyN), pp.112-117.

11. Alazzawi, L.K.; Elkateeb, AM.; Ramesh, A (2008) Scalability Analysis for Wireless Sensor Networks Routing Protocols. 22nd International Conference on Advanced Information Networking and Applications - Workshops (AINAW), pp.139-144.

12. Collotta, M.; Lo Bello, L.; Pau, G. (2015) A novel approach for dynamic traffic lights management based on Wireless Sensor Networks and multiple fuzzy logic controllers, Expert Systems with Applications, Vol. 42, Issue 13, pp. 5403-5415.

13. Huck, R.C.; Havlicek, J.P.; Sluss, J.J.; Stevenson, AR. (2005) A low-cost distributed control architecture for intelligent transportation systems deployment in the State of Oklahoma. IEEE Proceedings Intelligent Transportation Systems, pp.919-924.

14. 802.15.4: Wireless Medium Access Control (MAC) and Physical Layer (PHY) Specifications for Low-Rate Wireless Personal Area Networks (LR- WPANs) - June 2006 IEEE standard for information technology. Part 15.4.

15. Bluetooth Specification Version 4.0; Bluetooh SIG 2010.

16. Mandal, K.; Sen, A; Chakraborty, A; Roy, S.; Batabyal, S.; Bandyopadhyay, S. (2011) Road traffic congestion monitoring and measurement using active RFID and GSM technology, 14th International IEEE Conference on Intelligent Transportation Systems (ITSC), pp.1375-1379.

17. Zotos, N.; Stergiopoulos, C.; Anastasopoulos, K.; Bogdos, G.; Pallis, E.; Skianis, C. (2012) Case study of a dimmable outdoor lighting system with intelligent management and remote control, International Conference on Telecommunications and Multimedia (TEMU), pp.43-48.

18. Megalingam, Rajesh Kannan; Mohan, V.; Mohanan, A; Leons, P.; Shooja, R. (2010) Wireless Sensor Network for Vehicle Speed Monitoring and Traffic Routing System, 2nd International Conference on Mechanical and Electrical Technology (ICMET), pp.631-635.

19. Brahmi, H.I; Djahel, S.; Murphy, J. (2013) Improving emergency messages transmission delay in road monitoring based WSNs, 6th Joint IFIP Wireless and Mobile Networking Conference (WMNC), pp.1-8.

20. Collotta, M.; Pau, G.; Salerno, V.M.; Scatà, G. (2012) A Novel Road Monitoring Approach Using Wireless Sensor Networks, Sixth International Conference on Complex, Intelligent and Software Intensive Systems (CISIS), pp.376-381.

21. Collotta, M.; Messineo, A.; Nicolosi, G.; Pau, G. (2014) A self-powered Bluetooth Network for Intelligent Traffic light Junction Management, WSEAS Transactions on Information Science and Applications, Vol. 11, pp. 12-23.

22. Tsubota, T.; Bhaskar, A.; Chung, E.; Billot, R. (2011) Arterial traffic congestion analysis using Bluetooth Duration data, Australasian Transport Research Forum Proceedings, pp. 1-14.

23. Jie, L.; van Zuylen, H.; Chunhua, L.; Shoufeng, L. (2011) Monitoring travel times in an urban network using video, GPS and Bluetooth, Procedia of Social and Behavioral Sciences, pp. 630-637.

24. Araghi, B.N.; Christensen, L.T.; Lahrmann, H. (2013) Accurate Travel Time Estimation and Incident Detection Using Bluetooth and WiFi Technologies, Best Practices on Development of Monitoring Technologies and Services, VTT Technical Research Centre of Finland. 
25. Bachmann, C.; Roorda, M.J.; Abdulhai, B.; Moshiri, B. (2013) Fusing a Bluetooth Traffic Monitoring System With Loop Detector Data for Improved Freeway Traffic Speed Estimation, Intelligent Transportation Systems, Vol. 17, no. 2, pp. 153-164.

26. Collotta, M.; Pau, G.; Scatà, G. (2013) Deadline-aware scheduling perspectives in industrial wireless networks: A comparison between IEEE 802.15.4 and Bluetooth, International Journal of Distributed Sensor Networks, 2013, art. no. 602923.

27. Collotta, M.; Lo Bello, L.; Mirabella, O. (2007) Deadline-Aware Scheduling Policies for Bluetooth Networks in Industrial Communications, International Symposium on Industrial Embedded Systems (SIES), pp.156,163.

28. Collotta, M.; Lo Bello, L.; Mirabella, O. (2010) An innovative frequency hopping management mechanism for Bluetooth-based industrial networks, International Symposium on Industrial Embedded Systems (SIES), pp.45,50.

29. Horn, W. (1974) Some Simple Scheduling Algorithms, Naval Research Logistics Quaterly, 21.

30. OMNeT++: Discrete Event Simulator (2014), version 4.6, http://www.omnetpp.org/.

31. Network Simulator: NS-2 (2013), version 2.35, http://www.cs.uc.edu/ cdmc/ucbt/. 